DOI: 10.12957/demetra.2016.19730

\title{
A insegurança alimentar grave está associada a fatores de risco para doenças crônicas não transmissíveis e doença cardiovascular no Distrito Federal
}

\section{The severe food insecurity is associated with risk factors for non-transmissible chronic-disease and cardiovascular disease in Distrito Federal, Brazil}

Ana Lilian Bispo dos Santos'

Muriel Bavermann Gubert ${ }^{\dagger}$

Andressa Cristina Santos de Deus'

1 Universidade de Brasília,

Faculdade de Ciências da Saúde, Departamento de Nutrição, Programa de Pós-Graduação em

Saúde Coletiva. Brasília-DF, Brasil.

Correspondência / Correspondence

Ana Lilian Bispo dos Santos

E-mail: analilian_nutricao@yahoo.com.br

\section{Resumo}

Objetivo: Verificar a associação entre fatores de risco e ocorrência de doenças crônicas não transmissíveis (DCNT) com a insegurança alimentar grave (IAG) no Distrito Federal. Método: Trata-se de um estudo transversal, realizado com a população adulta do Distrito Federal. Os dados foram coletados por meio de questionário, que englobava perguntas socioeconômicas, consumo protetor e de risco para DCNT, ocorrência de DCNT e segurança alimentar aferida pela Escala Brasileira de Insegurança alimentar. A amostragem foi sistematizada por cotas, segundo renda e situação de IAG e segurança alimentar (SA). E composta por 324 adultos, dos quais $74,1 \%$ tinham segurança alimentar e 25,9\% insegurança alimentar grave. Resultados: Os resultados apontam uma associação positiva de IAG com doença cardiovascular (DCV) (OR:1,79), consumo de excesso de gordura provenientes de carnes vermelhas e pele de frango e maior risco de obesidade (OR: 2,21). Essa associação passa a ser negativa com consumo de alimentos protetores contra DCNT, como feijão (OR:0,47), frutas (OR:0,19) e hortaliças (OR:0,44). Indivíduos com IAG têm também menor chance de praticarem atividade física se comparados aos com SA (OR:0,45). Conclusão: Este estudo evidencia uma associação da IAG com DCV e maior prevalência de fatores de risco para DCNT. Esses achados são importantes para auxiliar no planejamento de políticas públicas de combate à fome, que devem ter como foco a promoção da saúde e redução da ocorrência de DCNT em população com IAG.

Palavras-chave: Segurança alimentar e nutricional. Fome. Doença crônica. Fatores de risco. 


\section{Abstract}

Objective: Determining the association between risk factors and the occurrence of non-communicable diseases (NCDs) with severe food insecurity (SFI) in the Distrito federal, Brazil. Method: This is a cross-sectional study, conducted with the adult population of the Federal District. The data were collected through a questionnaire. The questionnaire comprised socioeconomic questions, protective and risk food consumption for NCDs, the occurrence of NCDs and food security assessed by the Brazilian Scale of Food Insecurity. The sampling was processed by quotas in according to the income and food security status. The sample consisted of 324 adults, of whom $74.1 \%$ had food security (FS) and 25.9\% severe food insecurity. Results: The results show a positive association of SFI with cardiovascular disease (CVD) (OR: 1.79), the consumption of excess fat from red meat and chicken's skin and increased risk of obesity (OR: 2.21). This association becomes negative with consumption of protective foods such as beans (OR: 0.47 ), fruit (OR: 0.19) and vegetables (OR: 0.44). Individuals with SFI also have less chance to practice physical activity compared to those ones with FS (OR: 0.45). Conclusion: This study shows an association of SFI with CVD and higher prevalence of risk factors for NCDs. These findings are important to assist in the planning of public policies to combat hunger, which should focus on health promotion and reducing the occurrence of NCDs in the population with SFI.

Key words: Food security. Hunger. Chronic disease. Risk factors.

\section{Introdução}

A insegurança alimentar (IA) caracteriza-se pela falta de acesso regular e permanente a alimentos de qualidade, em quantidade suficiente, tendo como base práticas alimentares promotoras de saúde. ${ }^{1}$ Em 2013, a IA estava presente em 22,6\% de domicílios particulares brasileiros. ${ }^{2}$ Essa prevalência foi maior do que nos Estados Unidos, onde 14,0\% dos domicílios apresentavam IA, em 2014, ${ }^{3}$ porém semelhante a prevalências encontradas na África ${ }^{4} \mathrm{e}$ em regiões pobres da Austrália. ${ }^{5}$

A insegurança alimentar está relacionada à diminuição do consumo de alguns grupos de alimentos (frutas e hortaliças, cereais integrais, carnes, produtos lácteos), bem como redução na variedade e no tamanho das porções. ${ }^{6}$ Tais fatores podem originar inadequação da ingestão de nutrientes, trazendo impactos negativos na situação de saúde dos indivíduos, ${ }^{5}$ como carências nutricionais ${ }^{7}$ e doenças crônicas não transmissíveis (DCNT) ${ }^{8-10}$ entre elas, a obesidade. ${ }^{11}$ 
Estudos demonstram que a desnutrição pregressa, ocorrida durante a gestação e infância do indivíduo, é fator de risco para doenças crônicas não transmissíveis (DCNT). ${ }^{12}$ Há indícios, porém, que IA experimentada na fase adulta também pode estar associada às DCNT. ${ }^{9}$ Essa associação seria explicada por uma restrição qualitativa e quantitativa da dieta, ${ }^{13}$, com maior consumo de energia barata proveniente de alimentos de alta densidade calórica, ${ }^{14}$ resultando em um balanço energético positivo e desencadeando obesidade e outras doenças crônicas. ${ }^{5,9}$ Há evidencias de que dietas com consumo insuficiente de frutas e hortaliças e consumo excessivo de açúcares simples e de gordura saturada são importantes fatores de risco para DCNT.15,16

Estudo realizado na Austrália evidenciou associação entre IA e pior estado geral de saúde, maior necessidade de cuidados de saúde e depressão. ${ }^{5}$ Nos Estados Unidos, foi identificado maior prevalência de hiperlipidemia, hipertensão e diabetes entre indivíduos com IA quando comparados aos indivíduos que apresentavam segurança alimentar (SA). ${ }^{17}$ Segundo Gowda et al., ${ }^{8}$ a IA esteve associada à inflamação celular em estudo realizado com a população norte-americana. No Canadá, a IA implicou maior risco de doenças crônicas sensíveis à dieta. ${ }^{18} \mathrm{Na}$ Jordânia, pesquisadores identificaram a IA como fator associado a pior controle glicêmico em pacientes com diabetes tipo $2 .{ }^{19}$

Entretanto, no Brasil ainda são escassos os estudos que verificam a associação de IA e DCNT ou seus fatores de risco. Recentes estudos brasileiros constataram a associação entre IA e obesidade em mulheres e adolescentes do sexo feminino. ${ }^{20,21}$ Santos $^{22}$ pondera que ainda não há uma resposta definitiva sobre a relação fome e obesidade entre homens no Brasil.

Essa lacuna de conhecimento referente à associação entre IA e DCNT no Brasil merece ser melhor estudada. Pesquisar os aspectos relacionados a esse fenômeno é importante para o planejamento de futuras estratégias de intervenções nutricionais e de políticas públicas que garantam as condições de segurança alimentar da população, protegendo-a contra a ocorrência de DCNT. Dessa forma, o objetivo deste estudo foi pesquisar a associação entre fatores de risco e ocorrência de DCNT e IA grave (IAG) no Distrito Federal (DF).

\section{Método}

Trata-se de um estudo transversal, realizado com a população adulta do DF, no período de fevereiro a junho de 2014.

A amostragem foi por conveniência, sistemática e representativa, por cota para a população do DF em situação de SA e IAG. As cotas foram definidas por estratificação segundo faixa de renda domiciliar per capita e situação de segurança alimentar, abrangendo apenas indivíduos em situação de SA ou IAG. O tamanho estimado da amostra foi de 324 indivíduos, 240 em situação de SA e 84, de IAG, admitindo erro máximo amostral de $10 \%$ e intervalo de confiança igual a $95 \%$. 
A seleção dos entrevistados foi realizada no próprio dia da entrevista, que ocorreu em quatro pontos de grande fluxo de pessoas do DF: rodoviárias do Plano Piloto, de Taguatinga e da Ceilândia, bem como Estação Shopping do Metrô DF. A escolha desses pontos como local de coleta deu-se em virtude da maior possibilidade de preenchimento das cotas amostrais necessárias. Os indivíduos eram abordados sistematicamente após o termino de cada entrevista ou dispensa por não elegibilidade, sendo imediatamente selecionado o primeiro transeunte a passar pelo local da entrevista. O indivíduo escolhido respondia a um questionário de triagem, em que era verificada sua elegibilidade pelos critérios de inclusão/exclusão e de cota. Caso não fosse elegível, era dispensado, logo sendo convidado o próximo transeunte a passar pelo posto de coleta, e assim sucessivamente.

Foram adotados os seguintes critérios de inclusão: ter idade igual ou superior a 18 anos, residir no DF, ter conhecimento sobre a disponibilidade de alimentos no domicílio, estar em situação de SA ou IAG. Mulheres grávidas, pessoas com incapacidade cognitiva, surdos e mudos foram excluídos da amostra. Os indivíduos foram abordados nos locais de coleta e convidados a participar da pesquisa até que cada cota amostral fosse preenchida. Os pesquisadores permaneciam em local determinado pela administração dos locais, sendo os indivíduos abordados ao passarem por esta estação de coleta. A entrevista durava em torno de 10 minutos e era aplicada por pesquisadores treinados para tal.

Os dados foram coletados por meio da aplicação de questionário com perguntas fechadas. $\mathrm{O}$ questionário foi construído com o uso de dois módulos oriundos de pesquisas anteriores. $\mathrm{O}$ módulo sobre ocorrência de DCNT e seus fatores de risco foi o utilizado no Vigitel - Vigilância de Fatores de Risco e Proteção para Doenças Crônicas por Inquérito Telefônico ${ }^{23}$ - e o módulo de segurança alimentar contempla a Escala Brasileira de Insegurança Alimentar, utilizada na Pesquisa Nacional por Amostragem de Domicílios, em 2013.

O questionário foi testado previamente em estudo-piloto. E abordava os seguintes aspectos: a) características demográficas e socioeconômicas; b) padrão alimentar e atividade física (associados à ocorrência de DCNT); c) peso e altura referidos; d) frequência do consumo de bebidas alcoólicas; e) referência a diagnóstico médico anterior de DCNT (doença cardiovascular, diabetes e câncer); e f) situação de segurança alimentar.

Para verificar a ocorrência de DCNT, utilizou-se a variável de diagnóstico médico prévio de diabetes, doença cardiovascular (insuficiências cardíacas congestivas, acidente vascular cerebral, infarto agudo do miocárdio, arritmias cardíacas, isquemias, anginas, hipertensão arterial sistêmica) e câncer. ${ }^{23}$ Foram pesquisadas, como fatores de risco e de proteção para DCNT, as variáveis incluídas no Vigitel: $:^{23}$ excesso de peso (índice de massa corporal $\geq 25 \mathrm{~kg} / \mathrm{m}^{2}$ ) e obesidade (índice de massa corporal $\geq 30 \mathrm{~kg} / \mathrm{m}^{2}$ ); consumo de alimentos considerados marcadores de padrões saudáveis: feijão ( $\geq 1 \mathrm{x} /$ dia), fruta ( $\geq 3 \mathrm{x} /$ dia) e hortaliça ( $\geq 3 \mathrm{x} /$ dia); consumo de alimentos não saudáveis: carnes com excesso de gordura, leite com teor integral de gordura e refrigerante normal; prática de atividade física no tempo livre ( $\geq 150 \mathrm{~min}$./semana); consumo abusivo de bebidas alcoólicas (mulheres: $>4$ doses/ocasião e homens: >5doses/ocasião). 
Embora a obesidade seja considerada doença crônica, neste estudo ela foi avaliada como fator de risco para o desenvolvimento de outras DCNT relacionadas à dieta inadequada. ${ }^{24}$

Um escore de alimentação salutar foi calculado para avaliar as questões de consumo alimentar, incluindo as variáveis: feijão, frutas, verduras cruas, legumes cozidos, leite e refrigerante, em quatro níveis de frequência de consumo semanal (Quadro 1), segundo metodologia proposta por Souza et al. ${ }^{25} \mathrm{O}$ escore foi calculado a partir da soma da pontuação dos itens, podendo variar de 0 a 24 pontos.

Quadro 1. Descrição da pontuação utilizada para cálculo do escore de alimentação saudável.

\begin{tabular}{|c|c|c|c|c|c|}
\hline & $\mathbf{0}$ & 1 & 2 & 3 & 4 \\
\hline Feijão & $\begin{array}{c}\text { Nunca/ } \\
\text { quase nunca }\end{array}$ & $1-2 x /$ semana & $3-4 x /$ semana & $5-6 x /$ semana & Todos os dias \\
\hline Fruta & $\begin{array}{c}\text { Nunca/ } \\
\text { quase nunca }\end{array}$ & $1-2 x /$ semana & $3-4 x /$ semana & 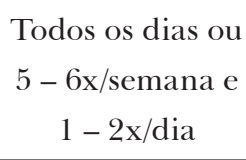 & $\begin{array}{l}\text { Todos os dias ou } \\
\begin{array}{c}5-6 x / \text { semana e } \\
\geq 3 x / \text { dia }\end{array}\end{array}$ \\
\hline Verdura crua & $\begin{array}{c}\text { Nunca/ } \\
\text { quase nunca }\end{array}$ & $1-2 x /$ semana & $3-4 x /$ semana & $\begin{array}{c}\text { Todos os dias ou } \\
5-6 x / \text { semana e } \\
1 x / \text { dia }\end{array}$ & $\begin{array}{c}\text { Todos os dias ou } \\
5-6 x / \text { semana } \mathrm{e} \\
\geq 2 \mathrm{x} / \text { dia }\end{array}$ \\
\hline $\begin{array}{l}\text { Legumes } \\
\text { cozidos }\end{array}$ & $\begin{array}{c}\text { Nunca/ } \\
\text { quase nunca }\end{array}$ & $1-2 x /$ semana & $3-4 x /$ semana & $\begin{array}{c}\text { Todos os dias ou } \\
5-6 x / \text { semana e } \\
\text { 1x/dia }\end{array}$ & $\begin{array}{c}\text { Todos os dias ou } \\
5-6 x / \text { semana e } \\
\geq 2 x / \text { dia }\end{array}$ \\
\hline Leite & $\begin{array}{c}\text { Nunca/ } \\
\text { quase nunca }\end{array}$ & $1-2 x /$ semana & $3-4 x /$ semana & $5-6 x /$ semana & Todos os dias \\
\hline Refrigerante & $\begin{array}{l}\text { Todos os } \\
\text { dias }\end{array}$ & $5-6 x /$ semana & $3-4 x /$ semana & $1-2 \mathrm{x} / \mathrm{semana}$ & $\begin{array}{c}\text { Nunca/quase } \\
\text { nunca e diet/light }\end{array}$ \\
\hline
\end{tabular}

Fonte: Souza Ade M, Bezerra IN, Cunha DB, Sichieri R. Evaluation of food intake markers in the Brazilian surveillance system for chronic diseases--VIGITEL (2007-2009). Rev Bras Epidemiol. 2011;14 Suppl 1:44-52.

As variáveis foram analisadas no programa SPSS Statistics versão 20. A descrição da amostra foi realizada por meio de análise univariada. As estimativas fornecidas pela análise bivariada de variáveis categóricas foram expressas por prevalências e intervalos de confiança de 95\%, sendo utilizado teste do qui-quadrado para comparação entre as variáveis categóricas. A análise ajustada foi feita por meio de regressão logística e expressa em odds ratio $(\mathrm{OR})$, sendo as variáveis consideradas no modelo para o ajuste biológico: sexo, idade e raça; e o ajuste social: escolaridade e renda. Para 
comparação das médias dos escores de alimentação saudável foi utilizado o teste t-student. O nível de significância considerado foi de $5 \%$, com intervalo de confiança de $95 \%$.

Todos os participantes da pesquisa foram informados sobre o objetivo e os procedimentos da pesquisa e que a participação era voluntária e anônima. E assinaram o Termo de Consentimento Livre e Esclarecido. Esta pesquisa foi aprovada pelo Comitê de Ética em Pesquisa da Universidade de Brasília sob o protocolo no 18340813.8 .0000 .0030$.

\section{Resultados}

A amostra final foi composta por 324 adultos moradores do DF, dos quais 74,1\% em situação de SA e 25,9\% em situação de IAG. A idade média foi de 39,1 (DP $\pm 15,9)$ anos e 53,7\% eram do sexo feminino. A DCNT mais prevalente foi doença cardiovascular (DCV) (21,9\%), seguida de diabetes (DM) $(9,0 \%)$ e câncer (CA) (3,4\%) (dados não apresentados em Tabelas).

Segundo as características sociodemográficas, indivíduos com SA apresentaram maiores níveis de escolaridade e renda que aqueles com IAG. Já em relação a fatores de risco e DCNT, indivíduos com IAG apresentaram maior prevalência de DCV, obesidade e consumo de pele de frango e menor prevalência de prática de atividade física, consumo de feijão, frutas e hortaliças quando comparados aos com SA $(\mathrm{p}<0,05)$.

Tabela 1. Características demográficas, de saúde, consumo alimentar e situação de segurança alimentar da população do Distrito Federal (n=324), 2014.

\begin{tabular}{lccccc}
\hline & \multicolumn{2}{c}{$\mathrm{SA}(\mathrm{n}=240)$} & \multicolumn{2}{c}{$\mathrm{IAG}(\mathrm{n}=84)$} & $\mathrm{P}$ \\
& $\mathrm{n}$ & $\%$ & $\mathrm{~N}$ & $\%$ & \\
\hline Sexo & & & & & \\
Feminino & 122 & 50,8 & 52 & 61,9 & 0,080 \\
Masculino & 118 & 49,2 & 32 & 38,1 & \\
Idade & & & & & \\
18 a 29 anos & 92 & 38,3 & 20 & 23,8 & 0,058 \\
30 a 39 anos & 42 & 17,5 & 19 & 22,6 & \\
40 a 49 anos & 45 & 18,8 & 25 & 29,8 & \\
50 a 59 anos & 29 & 12,1 & 12 & 14,3 & \\
$\geq 6$ anos & 32 & 13,3 & 8 & 9,5
\end{tabular}




\begin{tabular}{|c|c|c|c|c|c|}
\hline & \multicolumn{2}{|c|}{$\mathrm{SA}(\mathrm{n}=240)$} & \multicolumn{2}{|c|}{$\mathrm{IAG}(\mathrm{n}=84)$} & \multirow[t]{2}{*}{$\mathrm{P}$} \\
\hline & $\mathrm{n}$ & $\%$ & $\mathrm{~N}$ & $\%$ & \\
\hline \multicolumn{6}{|l|}{ Raça } \\
\hline Branca & 58 & 24,2 & 18 & 21,4 & 0,877 \\
\hline preta/parda & 174 & 72,5 & 63 & 75,0 & \\
\hline amarela/indigena & 8 & 3,3 & 3 & 3,6 & \\
\hline \multicolumn{6}{|c|}{ Escolaridade (anos de estudo) } \\
\hline$\leq 9$ anos & 58 & 24,2 & 36 & 42,9 & 0,003 \\
\hline$>9 e \leq 12$ anos & 118 & 49,2 & 35 & 41,7 & \\
\hline$>12$ anos & 64 & 26,6 & 13 & 15,4 & \\
\hline \multicolumn{6}{|l|}{ Renda per capita } \\
\hline$\leq 1 / 4 S M$ & 6 & 2,5 & 20 & 23,8 & $<0,00$ \\
\hline$>1 / 4$ a $1 / 2 S M$ & 32 & 13,3 & 24 & 28,6 & \\
\hline$>1 / 2$ a $1 S M$ & 60 & 25,0 & 23 & 27,4 & \\
\hline$>1 a 2 S M$ & 77 & 32,1 & 11 & 13,1 & \\
\hline$>2 S M$ & 65 & 27,1 & 6 & 7,1 & \\
\hline
\end{tabular}

\section{Padrão alimentar}

consumo protetor

feijão $\geq 1 \mathrm{x} / \mathrm{dia}$

168

70,0

44

52,4

0,003

fruta $\geq 3 \mathrm{x} / \mathrm{dia}$

28

11,7

2

2,4

0,012

hortaliças $\geq 3 \mathrm{x} / \mathrm{dia}$

61

25,4

11

13,1

0,019

consumo de risco

leite integral

140

58,3

46

54,8

0,569

refrigerante normal

120

gordura da carne vermelha

69

50,0

40

47,6

0,707

pele do frango

50

28,8

33

39,3

0,074

\section{Estado nutricional}

sobrepeso $\left(I M C \geq 25 \mathrm{~kg} / \mathrm{m}^{2}\right)$

86

35,8

25

29,8

0,313

obesidade $\left(I M C \geq 30 \mathrm{~kg} / \mathrm{m}^{2}\right)$

28

11,7

19

22,6

0,014 


\begin{tabular}{|c|c|c|c|c|c|}
\hline & \multicolumn{2}{|c|}{$\mathrm{SA}(\mathrm{n}=240)$} & \multicolumn{2}{|c|}{$\mathrm{IAG}(\mathrm{n}=84)$} & \multirow[t]{2}{*}{$\mathrm{P}$} \\
\hline & $\mathrm{n}$ & $\%$ & $\mathrm{~N}$ & $\%$ & \\
\hline \multicolumn{6}{|l|}{ Atividade física } \\
\hline recomendado $(\geq 150 \mathrm{~min} / \mathrm{semana})$ & 83 & 34,6 & 16 & 19,0 & 0,008 \\
\hline \multicolumn{6}{|l|}{ Consumo de bebida alcoólica } \\
\hline uso abusivo* & 65 & 27,1 & 17 & 20,2 & 0,214 \\
\hline \multicolumn{6}{|l|}{$\begin{array}{l}\text { Doenças crônicas não } \\
\text { transmissíveis }\end{array}$} \\
\hline Doença Cardiovascular (DCV) & 46 & 19,2 & 25 & 29,8 & 0,043 \\
\hline Diabetes & 20 & 8,3 & 9 & 10,7 & 0,511 \\
\hline Câncer & 6 & 2,5 & 5 & 6,0 & 0,133 \\
\hline
\end{tabular}

Salário mínimo (SM) na época da pesquisa $=\mathrm{R} \$ 724,00 ; *$ (+4 doses/ocasião) e ô (+5 doses/ocasião); doença cardiovascular (DCV): insuficiências cardíacas congestivas, acidente vascular cerebral, infarto agudo do miocárdio, arritmias cardíacas, isquemias, anginas, hipertensão arterial sistêmica.

A Tabela 2 apresenta as OR para ocorrência de DCNT segundo situação de SA. Indivíduos com IAG possuem $79 \%$ mais chance de ter DCV que indivíduos com SA. Porém quando essas variáveis são controladas por fatores biológicos ou sociais no modelo de regressão, essa associação deixa de ser significativa.

Na avaliação do consumo alimentar segundo índice de alimentação saudável, em uma escala com escore máximo de 24 pontos, a média do escore de alimentação saudável entre indivíduos com SA foi de 15,8 (DP $\pm 4,4)$ pontos e de 12,3 (DP $\pm 3,9)$ pontos nos indivíduos com IAG ( $<<0,001)$ (dados não apresentados em Tabelas).

Houve associação entre IAG e menor consumo de alimentos considerados protetores contra DCNT, como feijão (OR 0,47; IC 95\% 0,28-0,79), frutas (OR 0,19; IC 95\% 0,04-0,79), hortaliças (OR 0,44; IC 95\%: 0,22-0,89), e maior consumo de pele de frango (OR 2,11; IC95\% 1,23-3,64). Essas associações se mantêm mesmo quando as variáveis biológicas e sociais são controladas. Adultos com IAG tendem também a consumir mais carne vermelha com gordura do que os em SA, quando o modelo é ajustado por variáveis socioeconômicas .(Tabela 2)

Em relação ao estado nutricional (Tabela 2), indivíduos com IAG têm 2,21 vezes mais chances de ser obesos que os com SA, porém essa associação não permanece após ajustes por regressão. Adultos com IAG também têm menor chance de ser fisicamente ativos comparados àqueles com SA, mesmo quando essas variáveis são controladas por fatores biológicos no modelo. 
Tabela 2. Prevalências, OR brutos e ajustados (por fatores biológicos e sociais) para associação entre insegurança alimentar grave (IAG) e Doenças crônicas não transmissíveis (DCNT), consumo de alimentos protetores e de risco para DCNT, estado nutricional, atividade e consumo de bebida alcoólica. Distrito Federal, 2014.

\begin{tabular}{|c|c|c|c|c|c|}
\hline & $\begin{array}{c}\text { Prev. } \\
(\%) \\
\text { IAG }\end{array}$ & $\begin{array}{c}\text { Prev. } \\
(\%) \\
\text { SA }\end{array}$ & $\begin{array}{l}\text { OR bruto } \\
(95 \% \mathrm{IC})\end{array}$ & $\begin{array}{l}\text { OR aj. bio. } \\
(95 \% \text { IC) }\end{array}$ & $\begin{array}{l}\text { OR aj. soc. } \\
(95 \% \text { IC) }\end{array}$ \\
\hline \multicolumn{6}{|l|}{ DCNT } \\
\hline DCV & 29,8 & 19,2 & $1,79(1,01-3,15)^{*}$ & $1,87(0,97-3,61)$ & $1,52(0,79-2,95)$ \\
\hline Diabetes & 10,7 & 8,3 & $1,32(0,58-3,03)$ & $1,28(0,50-3,26)$ & $0,94(0,35-2,49)$ \\
\hline Câncer & 6,0 & 2,5 & 2,47 (0,73-8,31) & 2,23 (0,63-7,87) & $2,57(0,63-10,58)$ \\
\hline \multicolumn{6}{|l|}{ Consumo protetor } \\
\hline Feijão & 52,4 & 70,0 & $0,47(0,28-0,79)^{*}$ & $0,47(0,28-0,81)^{*}$ & $0,41(0,23-0,73) *$ \\
\hline Fruta & 2,4 & 11,7 & $0,19(0,04-0,79)^{*}$ & $0,18(0,04-0,77)^{*}$ & $0,19(0,04-0,90)^{*}$ \\
\hline Hortaliça & 13,1 & 25,4 & $0,44(0,22-0,89)^{*}$ & $0,39(0,19-0,79)^{*}$ & $0,43(0,20-0,93) *$ \\
\hline \multicolumn{6}{|l|}{ Consumo de risco } \\
\hline Leite integral & 54,8 & 58,3 & $0,87(0,52-1,43)$ & $0,89(0,53-1,49)$ & $0,56(0,31-1,01)$ \\
\hline Refrigerante normal & 47,6 & 50,0 & $0,91(0,55-1,50)$ & $0,95(0,56-1,62)$ & $0,90(0,51-1,59)$ \\
\hline $\begin{array}{l}\text { Gordura da carne } \\
\text { vermelha }\end{array}$ & 39,3 & 28,8 & $1,60(0,95-2,70)$ & $1,71(0,99-2,94)$ & $1,83(1,01-3,31)^{*}$ \\
\hline Pele do frango & 35,7 & 20,8 & $2,11(1,23-3,64)^{*}$ & $2,17(1,23-3,81)^{*}$ & $1,89(1,02-3,50)^{*}$ \\
\hline \multicolumn{6}{|l|}{ Estado nutricional } \\
\hline Sobrepeso & 29,8 & 35,8 & $0,76(0,44-1,30)$ & $0,64(0,37-1,13)$ & $0,76(0,44-1,30)$ \\
\hline Obesidade & 22,6 & 11,7 & $2,21(1,16-4,22)^{*}$ & $1,86(0,95-3,63)$ & $1,71(0,79-3,70)$ \\
\hline \multicolumn{6}{|l|}{ Atividade física } \\
\hline $\begin{array}{l}\text { Recomendado ( } \geq 150 \\
\mathrm{~min} / \mathrm{semana})\end{array}$ & 19,0 & 34,6 & $0,45(0,24-0,82)^{*}$ & $0,47(0,25-0,87)^{*}$ & $0,73(0,37-1,44)$ \\
\hline \multicolumn{6}{|l|}{ Bebida alcoólica } \\
\hline Consumo abusivo & 20,2 & 27,1 & $0,68(0,37-1,25)$ & $0,78(0,42-1,47)$ & $0,84(0,44-1,64)$ \\
\hline
\end{tabular}

OR - categoria de referência SA. OR aj. bio. (OR ajustado para fatores biológicos): odds ratio ajustado para sexo, idade e raça; OR aj. soc. (OR ajustado para fatores sociais): odds ratio ajustado para escolaridade e renda; IC: intervalo de confiança; *p<0,05. 


\section{Discussão}

Como esperado, indivíduos com IAG apresentaram menor escolaridade e renda quando comparados aos com SA. ${ }^{2}$ Essa maior vulnerabilidade econômica pode estar relacionada a um dispêndio alimentar inadequado, como evidenciado em estudos anteriores. ${ }^{26,27}$ Indivíduos em situação de pobreza tendem a ter uma dieta mais barata e nutricionalmente mais pobre, com maior consumo de alimentos com alta densidade calórica e baixo valor nutricional, bem como com menor de alimentos saudáveis. ${ }^{26,27}$ No presente estudo, pessoas com IAG consumiram menos frutas, feijões e hortaliças, ao mesmo tempo que comeram mais carnes gordurosas.

Dados do Vigitel Brasil 2014 apontam que no DF os percentuais de consumo regular de frutas e hortaliças, bem como do de carnes com excesso de gordura, na população do DF, foram de $41,6 \%$ e 29,3\%, respectivamente. ${ }^{23}$ Infelizmente, o Vigitel não permite verificar o consumo entre pessoas com IAG ou SA. Na presente pesquisa, para adultos com IAG, os percentuais de consumo foram de 2,4\% (frutas), 13,1\% (hortaliças), 39,3\% (gordura da carne vermelha) e 35,7\% (pele de frango). Isso evidencia um pior padrão alimentar dos indivíduos com IAG quando comparados ao consumo da população do DF como um todo.

Observou-se que indivíduos com IAG têm mais chances de apresentar DCV comparados àqueles com SA. Ainda não são claros os mecanismos pelos quais a insegurança alimentar está relacionada a uma maior ocorrência de DCNT. ${ }^{9}$ Essa associação está bem documentada em relação à diabetes, mas carece de maiores estudos quanto às demais. ${ }^{17}$ Essa associação entre IA e DCNT parece estar mediada por inadequação do consumo alimentar com dependência de alimentos energeticamente densos e baratos ${ }^{9,28}$ e com o sobrepeso e obesidade presente nessa população. ${ }^{20,21,29}$ A população em situação de IAG vivencia a chamada dupla-carga de doenças. Ao mesmo tempo que esta população pode ser afetada por carências de micronutrientes, déficits nutricionais e doenças infecciosas, pode ser também afetada pelas DCNT e obesidade. ${ }^{30,31}$ Sendo assim, ligações entre pobreza e DCNT devem ser consideradas na formulação de políticas públicas de combate à fome. ${ }^{32}$

A atividade física regular é sabidamente fator protetor contra DCNT. ${ }^{33}$ No Brasil, a frequência de adultos que praticam atividades físicas no tempo livre, equivalentes a pelo menos 150 minutos por semana, varia entre 30,4\% em São Paulo e 47,1\% em Florianópolis. Em Brasília, para a população como um todo, essa prática foi de $37,4 \% .{ }^{23}$ Nesta pesquisa, essa frequência foi de $19,0 \%$ entre os indivíduos com IAG. Adultos em situação de IAG têm 55\% menos chances de praticar atividade física que os de SA. Essa associação permanece quando ajustada para variáveis biológicas, porém deixa de ser significativa quando as variáveis sociais são controladas no modelo. Estudos anteriores evidenciaram que indivíduos menos favorecidos economicamente têm menos acesso e praticam menos atividade física que aqueles de classe social mais favorecida. ${ }^{34-36}$ A pobreza, presente na maioria dos casos de IAG, contribui para exclusão social da prática de atividade física. Nesse contexto, o ambiente favorece o comportamento de risco para DCNT, tratando-se de população com IAG. ${ }^{34-36}$ 


\section{Conclusão}

Os resultados deste trabalho indicam uma tendência de associação positiva da IAG com DCV, maior consumo de carnes com excesso de gordura e obesidade. Essa associação passa a ser negativa para as variáveis relacionadas a comportamento protetor contra DCNT, como a prática de atividade física, consumo de feijão, frutas e hortaliças.

Grupos economicamente menos privilegiados apresentam elevadas prevalências de DCNT e IAG. É de fundamental importância entender a associação entre essas variáveis no sentido de auxiliar no planejamento de políticas públicas mais eficientes para manutenção das condições de saúde e segurança alimentar desses indivíduos, promovendo a redução da fome sem contribuir para o incremento das DCNT no país.

\section{Referências}

1. Brasil. Lei no 11.346 , de 15 de setembro de 2006. Cria o Sistema Nacional de Segurança Alimentar e Nutricional - SISAN com vistas em assegurar o direito humano à alimentação adequada e dá outras providências. Diário Oficial da União 18 set. 2006.

2. Instituto Brasileiro de Geografia e Estatística. Pesquisa Nacional por Amostra de Domicílio: segurança alimentar 2013. Rio de Janeiro: IBGE; 2014.

3. Coleman-Jensen A, Rabbitt MP, Gregory C, Singh A. Household food security in the United States in 2014: USDA, Economic Research Service. Washington: USDA; 2015.

4. United Nations. Food and Agriculture Organization. The state of food insecurity in the world: how does international price volatility affect domestic economies and food security. Canada: FAO; 2011.

5. Ramsey R, Giskes K, Turrell G, Gallegos D. Food insecurity among adults residing in disadvantaged urban areas: potential health and dietary consequences. Public Health Nutr. 2012; 15(2):227-37.

6. Panigassi G, Segall-Corrêa AM, Marin-León L, Pérez-Escamilla R, Maranha KL, Sampaio MFA. Insegurança alimentar intrafamiliar e perfil de consumo de alimentos. Rev Nutr. 2008; 21(Supl.):135s144s.

7. Kirkpatrick SI, Dodd KW, Parsons R, Ng C, Garriguet D, Tarasuk V. Household food insecurity is a stronger marker of adequacy of nutrient intakes among canadian compared to american youth and adults. J Nutr. 2015; 145(7):1596-603.

8. Gowda C, Hadley C, Aiello AE. The association between food insecurity and inflammation in the US adult population. Am J Public Health 2012; 102(8):1579-86.

9. Laraia BA. Food insecurity and chronic disease. Adv Nutr. 2013; 4(2):203-12.

10. Shariff ZM, Sulaiman N, Jalil RA, Yen WC, Yaw YH, Taib MN, et al. Food insecurity and the metabolic syndrome among women from low income communities in Malaysia. Asia Pac J Clin Nutr. 2014; 23(1):138-47. 
11. Velasquez-Melendez G, Schlussel MM, Brito AS, Silva AA, Lopes-Filho JD, Kac G. Mild but not light or severe food insecurity is associated with obesity among Brazilian women. J Nutr. 2011; 141(5):898-902.

12. Langley-Evans SC, McMullen S. Developmental origins of adult disease. Med Princ Pract. 2010; 19(2):87-98.

13. Nguyen BT, Shuval K, Bertmann F, Yaroch AL. The Supplemental Nutrition Assistance Program, Food Insecurity, Dietary Quality, and Obesity Among U.S. Adults. Am J Public Health 2015; 105(7):1453-9.

14. Bem Lignani J, Sichieri R, Burlandy L, Salles-Costa R. Changes in food consumption among the Programa Bolsa Familia participant families in Brazil. Public Health Nutr. 2011; 14(5):785-92.

15. Moura EC, Silva SA, Malta DC, Morais Neto OL. Risk and protective factors for chronic noncommunicable diseases: the VIGITEL telephone disease surveillance system, Brazil, 2007. Cad Saude Publica 2011; 27(3):486-96.

16. World Health Organization. Global status report on non communicable diseases 2010. Geneva: WHO; 2011.

17. Seligman HK, Laraia BA, Kushel MB. Food insecurity is associated with chronic disease among low-income NHANES participants. J Nutr. 2010; 140(2):304-10.

18. Egeland GM, Johnson-Down L, Cao ZR, Sheikh N, Weiler H. Food insecurity and nutrition transition combine to affect nutrient intakes in Canadian arctic communities. J Nutr. 2011; 141(9):1746-53.

19. Bawadi HA, Ammari F, Abu-Jamous D, Khader YS, Bataineh S, Tayyem RF. Food insecurity is related to glycemic control deterioration in patients with type 2 diabetes. Clin Nutr. 2012; 31(2):250-4.

20. Kac G, Velasquez-Melendez G, Schlussel MM, Segall-Correa AM, Silva AA, Perez-Escamilla R. Severe food insecurity is associated with obesity among Brazilian adolescent females. Public Health Nutr. 2012; 15(10):1854-60.

21. Schlussel MM, Silva AA, Perez-Escamilla R, Kac G. Household food insecurity and excess weight/ obesity among Brazilian women and children: a life-course approach. Cad Saude Publica 2013; 29(2):219-26.

22. Santos LM. Obesity, poverty, and food insecurity in Brazilian males and females. Cad Saude Publica 2013; 29(2):237-9.

23. Brasil. Ministério da Saúde. Vigitel Brasil 2013: vigilância de fatores de risco e proteção para doenças crônicas por inquérito telefônico. Brasilia: Ministério da Saúde; 2014.

24. World Health Organization. Global status report on non communicable diseases 2010. Geneva: World Health Organization; 2011. 162 p.

25. Souza AM, Bezerra IN, Cunha DB, Sichieri R. Evaluation of food intake markers in the Brazilian surveillance system for chronic diseases--VIGITEL (2007-2009). Rev Bras Epidemiol. 2011; 14(Suppl 1):44-52.

26. Cotta RM, Machado JC. The Bolsa Familia cash transfer program and food and nutrition security in Brazil: a critical review of the literature. Rev Panam Salud Publica 2013; 33(1):54-60. 
27. Sartori AGO. Consumo alimentar de beneficiários do Programa Bolsa Família [dissertação]. Piracicaba (SP): Universidade de São Paulo; 2013.

28. Hanson KL, Connor LM. Food insecurity and dietary quality in US adults and children: a systematic review. Am J Clin Nutr. 2014; 100(2):684-92.

29. Conde WL, Monteiro CA. Nutrition transition and double burden of undernutrition and excess of weight in Brazil. Am J Clin Nutr. 2014; 100(6):1617S-22S.

30. Schramm JMA, Oliveira AF, Leite IC, Valente JG, Gadelha AMJ, Portela MC, et al. Epidemiological transition and the study of burden of disease in Brazil. Ciênc Saúde Coletiva 2004; 9(4):897-908.

31. Gubert MB, Spaniol AM, Bortolini GA, Perez-Escamilla R. Household food insecurity, nutritional status and morbidity in Brazilian children. Public Health Nutr. 2016; 19(12):2240-45.

32. Seligman HK, Schillinger D. Hunger and socioeconomic disparities in chronic disease. N Engl J Med. 2010; 363(1):6-9.

33. Barreto SM, Pinheiro ARO, Sichieri R, Monteiro CA, Batista Filho M, Schimidt MI, et al. Análise da estratégia global para alimentação, atividade física e saúde, da Organização Mundial da Saúde. Epidemiol Serv Saúde 2005; 14(1):41-68.

34. Florindo AA, Salvador EP, Reis RS, Guimaraes VV. Perception of the environment and practice of physical activity by adults in a low socioeconomic area. Rev Saude Publica 2011; 45(2):302-10.

35. Gomes CS, Matozinhos FP, Mendes LL, Pessoa MC, Velasquez-Melendez G. Physical and social environment are associated to leisure time physical activity in adults of a brazilian city: a cross-sectional study. PLoS One 2016; 11(2):e0150017.

36. Florindo AA, Salvador EP, Reis RS. Physical activity and its relationship with perceived environment among adults living in a region of low socioeconomic level. J Phys Act Health 2013; 10(4):563-71. 
